\title{
Structure of Arabidopsis thaliana 5-methylthioribose kinase reveals a more occluded active site than its bacterial homolog Shao-Yang Ku ${ }^{1,2}$, Kenneth A Cornell ${ }^{3}$ and P Lynne Howell ${ }^{* 1,2}$
}

\author{
Address: ${ }^{1}$ Program in Molecular Structure and Function, Research Institute, Hospital for Sick Children, 555 University Avenue, Toronto, Ontario, \\ M5G 1X8, CANADA, ${ }^{2}$ Department of Biochemistry, Faculty of Medicine, University of Toronto, Medical Science Building, Toronto, Ontario, M5S \\ 1A8, CANADA and ${ }^{3}$ Department of Chemistry and Biochemistry, Boise State University, 1910 University Drive, Boise, Idaho 83725-1520, USA \\ Email: Shao-Yang Ku - syku@sickkids.ca; Kenneth A Cornell - kencornell@boisestate.edu; P Lynne Howell* - howell@sickkids.ca \\ * Corresponding author
}

Published: 25 October 2007

BMC Structural Biology 2007, 7:70 doi:10.1 186/1472-6807-7-70

This article is available from: http://www.biomedcentral.com/l472-6807/7/70

(c) $2007 \mathrm{Ku}$ et al; licensee BioMed Central Ltd.

This is an Open Access article distributed under the terms of the Creative Commons Attribution License (http://creativecommons.org/licenses/by/2.0), which permits unrestricted use, distribution, and reproduction in any medium, provided the original work is properly cited.
Received: 4 July 2007

Accepted: 25 October 2007

\begin{abstract}
Background: Metabolic variations exist between the methionine salvage pathway of humans and a number of plants and microbial pathogens. 5-Methylthioribose (MTR) kinase is a key enzyme required for methionine salvage in plants and many bacteria. The absence of a mammalian homolog suggests that MTR kinase is a good target for the design of specific herbicides or antibiotics.

Results: The structure of Arabidopsis thaliana MTR kinase co-crystallized with ATP $\gamma$ and MTR has been determined at I.9 ^ resolution. The structure is similar to B. subtilis MTR kinase and has the same protein kinase fold observed in other evolutionarily related protein kinase-like phosphotransferases. The active site is comparable between the two enzymes with the DXE-motif coordinating the nucleotide-Mg, the D238 of the HGD catalytic loop polarizing the MTR OI oxygen, and the RR-motif interacting with the substrate MTR. Unlike its bacterial homolog, however, the Gly-rich loop (G-loop) of A. thaliana MTR kinase has an extended conformation, which shields most of the active site from solvent, a feature that resembles eukaryotic protein kinases more than the bacterial enzyme. The G- and W-loops of $A$. thaliana and B. subtilis MTR kinase adopt different conformations despite high sequence similarity. The ATP $\gamma S$ analog was hydrolyzed during the co-crystallization procedure, resulting in ADP in the active site. This suggests that the A. thaliana enzyme, like its bacterial homolog, may have significant ATPase activity in the absence of MTR.
\end{abstract}

Conclusion: The structure of $A$. thaliana MTR kinase provides a template for structure-based design of agrochemicals, particularly herbicides whose effectiveness could be regulated by nutrient levels. Features of the MTR binding site offer an opportunity for a simple organic salt of an MTR analog to specifically inhibit MTR kinase.

\section{Background}

S-adenosyl-L-methionine (SAM) is an important metabolite in plants as it not only provides the methyl group required for SAM-dependent biomethylation reactions, but also acts as the precursor for the biosynthesis of polyamines, nicotianamine, phytosiderophores, and the plant hormone ethylene [1]. SAM is metabolized to S-adenosylhomocysteine (SAH) in biological methylation, and to 5'-methylthioadenosine (MTA) in many other essential biological processes. While SAH is the product and a 
potent inhibitor of all SAM-dependent methyltransferases [2], MTA inhibits spermine synthase [3] in polyamine biosynthesis, and 1-aminocyclopropane-1-carboxylic acid synthase [4] in ethylene biosynthesis. Both polyamine and ethylene are important in plant development, growth, stress response and survival [5-7]. To promptly remove the toxic by-product MTA and to ensure sufficient supply of methionine and SAM, a methionine salvage pathway that recycles the methylthio moiety of MTA to methionine has evolved in almost all organisms. In plants, this pathway is also known as the Yang cycle [8]. In this cycle MTA is first depurinated to 5-methylthioribose (MTR), and then phosphorylated to form MTR 1phosphate (MTR 1-P). MTR 1-P then undergoes isomerization, dehydration, enolization and dephosphorylation to form the acireductone, 1,2-dihydroxyl-3-keto-5-methylthiopentene. This acireductone is subsequently converted to 2-keto-4-methylthiobutyrate and then transaminated to methionine $[8,9]$.

Metabolic variation in how MTA is recycled to methionine exists between different species. In bacteria and plant cells, two enzymes, MTA nucleosidase and MTR kinase, are required to convert MTA to MTR-1-P while in mammalian cells, MTA phosphorylase [10] phosphorylizes MTA to MTR 1-P in a single step. This metabolic difference has been explored and analogs of MTA and MTR have been synthesized for the development of specific antibiotics and herbicides [11-14]. MTR kinase is not an essential enzyme for the organisms' survival if methionine is present in the growth medium [15], but in the absence of methionine, expression of MTR kinase will prompt an organism to uptake MTA and/or MTR from the environment to replenish its sulfur pool $[15,16]$. Interestingly, even when sulfur is not depleted in the environment, numerous analogs of MTR have demonstrated selective growth inhibition of MTR kinase-containing bacteria $[11,12]$. Not surprisingly, the bactericidal activity of these MTR analogs, particularly trifluoromethylthioribose, is enhanced if the de novo methionine synthesis pathway is also blocked [17]. In plants, the expression of MTR kinase is also up-regulated under sulfur-limiting conditions, although the expression is not correlated with ethylene biosynthesis $[15,16,18]$. While most studies of MTR analogs have been performed on bacterial MTR kinases for the design of novel antibiotics, similar principles can be applied to the plant system to develop new herbicide and/ or herbicide-resistant transgenic crops that would improve agriculture efficiency.

The modern rational approach to new agrochemical discovery includes structure-based design approaches [19], which requires a detailed understanding of the target enzyme's structure, catalytic mechanism and substrate specificity. To this end, we present the first structural anal- ysis of A. thaliana MTR kinase in complex with ADP and MTR.

\section{Results and Discussion Overall fold of MTR kinase}

The structure of Arabidopsis thaliana MTR kinase has two lobes: a smaller N-lobe and a larger C-lobe connected by a linker region (residues 117-123) (Fig. 1 and 2a). The Nlobe is composed of five anti-parallel $\beta$ strands $(\beta 1-\beta 5)$ flanked by three short $\alpha$ helices $(\alpha 1 \mathrm{~A}, \alpha 2 \mathrm{~B}$ and $\alpha 2)$ in an $\alpha \alpha \beta \beta \beta \alpha \beta \beta$ topology (Fig. 2a). Helices $\alpha 1 \mathrm{~A}$ and $\alpha 1 \mathrm{~B}$ are located at the N-terminus, while helix $\alpha 2$ is inserted between strands $\beta 3$ and $\beta 4$ and lies beneath the $\beta$ strands near the C-lobe (Fig. 2a). The larger C-lobe is predominantly $\alpha$ helical with twelve $\alpha$ helices ( $\alpha 3-14)$ and six short $3_{10}$ helices $(\eta A-B, \eta 1$ and $\eta 3-5)$ along with four short $\beta$ strands $(\beta 7-\beta 10)$ between helices $\alpha 8$ and $\alpha 9$, and a short strand, $\beta 6$, at the C-terminal end of the linker region. Strand $\beta 8$ forms an anti-parallel $\beta$ turn with strand $\beta 9$, while strands $\beta 7$ and $\beta 10$ form a second anti-parallel sheet. The bilobal structural organization as well as the topology profile of MTR kinase is consistent with that found in eukaryotic protein kinases.

A. thaliana MTR kinase has 35\% amino acid sequence identity with Bacillus subtilis MTR kinase. The two enzymes share a similar secondary structure profile (Fig. 1) [20] and have a $\mathrm{C}^{\alpha}$ root mean square deviation (RMSD) of 1.7 $\AA$ (Fig. 3a). MTR kinase is structurally similar to other evolutionarily related protein kinase-like phosphotransferases. A comparison of the structure of A. thaliana MTR kinase to other known structures using Dali reveals that the enzyme resembles choline kinase (PDB:1NW1) [21], APH Type IIIa (APH(3')-IIIa) (PDB:1J7I) [22], and the archaeal atypical serine protein kinase Rio2 (PDB:1ZAR) [23] with Z-scores of 14.4, 12.5 and 11, $\mathrm{C}^{\alpha}$ RMSD's of 4.8 $\AA$, $4.6 \AA$ and $3.6 \AA$, and aligned sequence identities of $14 \%, 13 \%$ and $19 \%$, respectively [20].

\section{Quaternary structure}

The structure of $A$. thaliana MTR kinase has two subunits in the asymmetric unit related by a non-crystallographic 2 -fold (Fig. 2b). Approximately $1300 \AA^{2}$ or $6.7 \%$ of the surface area of each monomer is buried at the dimer interface [24]. Even though the plant enzyme underwent a complex multi-step refolding process [25], its dimeric structure resembles that of the bacterial enzyme [26]. Size exclusion chromatography of $A$. thaliana MTR kinase (data not shown), the physical characterization of other plant MTR kinases [27] as well as the apparent cooperative kinetics observed for the rice enzyme [15] all suggest that MTR kinase functions as a homodimer. The inter-subunit interactions in the dimer interface of A. thaliana MTR kinase are predominantly symmetrical and are concentrated at the C-terminus of helix $\alpha 8$ (Residues K217, 


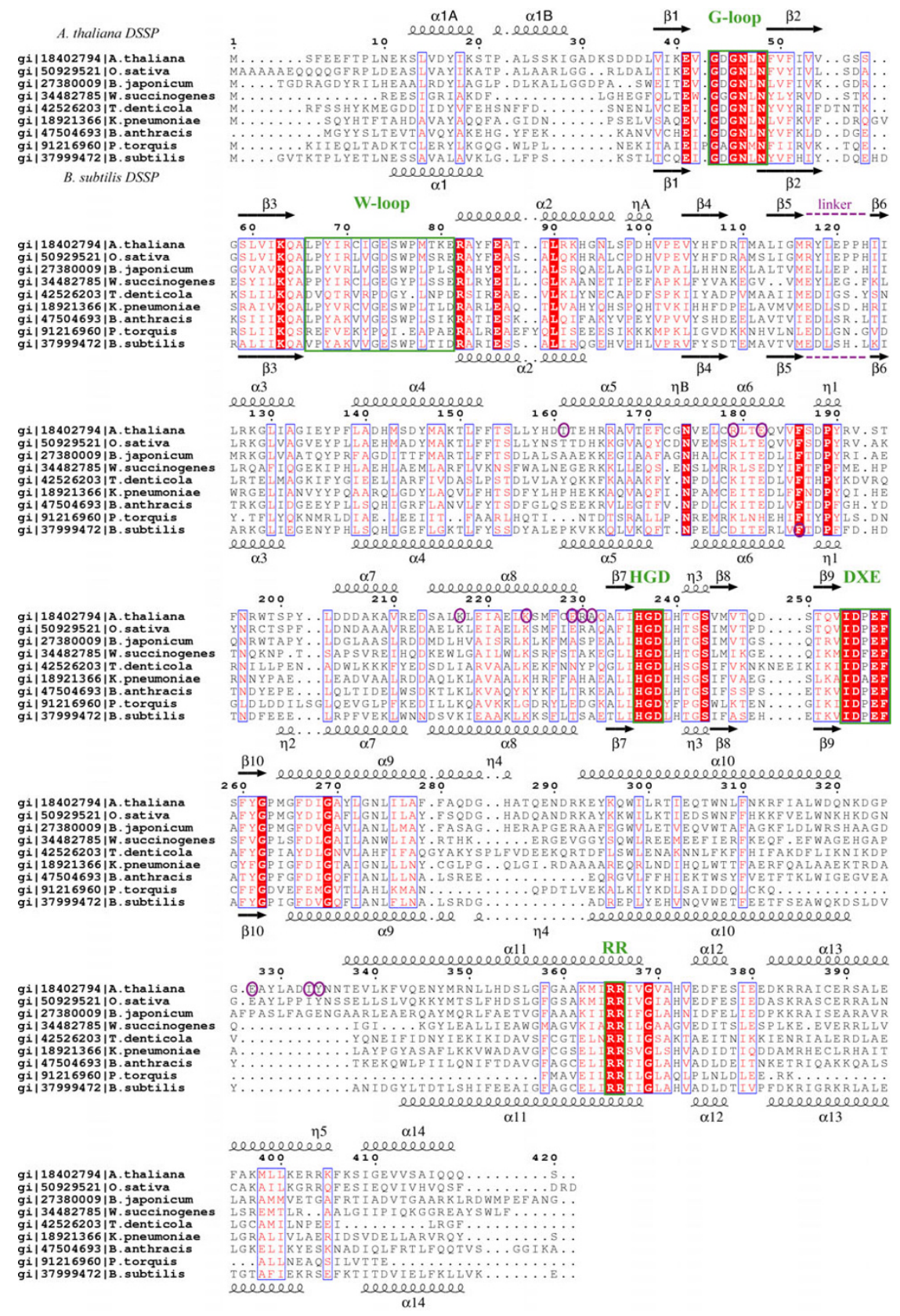

\section{Figure I}

Multiple sequence alignment of selected MTR kinase sequences mapping the secondary structural elements found in A. thaliana (top) and B. subtilis (bottom) MTR kinase structures. The protein sequences aligned are from Arabidopsis thaliana, Oryza sativa, Bradyrhizobium japonicum, Wolinella succinogenes, Treponema denticola, Klebsiella pneumoniae, Bacillus anthracis, Psychroflexus torquis and Bacillus subtilis. Their gene identifiers (gi) from the National Center for Biotechnology Information (NCBI) are also listed. The secondary structural elements were defined according to DSSP [48], numbered by the order of their appearance, and named to be consistent between the two enzymes. $\alpha$ helices are presented as curly lines and $\beta$ strands by arrows. $\eta$ stands for a $3_{10}$ helix. According to DSSP, the $A$. thaliana enzyme does not have the $\eta 2$ helix found in the $B$. subtilis enzyme. The multiple sequence alignment was performed using the program T-Coffee [49], and the figure prepared using ESPript [50]. Strictly conserved residues are denoted in white and framed in blue boxes with a red background; residues conserved in at least $70 \%$ of the sequences are denoted in red and framed in blue boxes with a white background. The G-loop, the W-loop, the Mg-binding DXE-motif, the HGD catalytic loop, and the MTR-binding RR-motif are framed in green boxes. Residues circled in purple are involved in dimer formation. The linker region connecting the $\mathrm{N}$-lobe and $\mathrm{C}$-lobe is also indicated. 

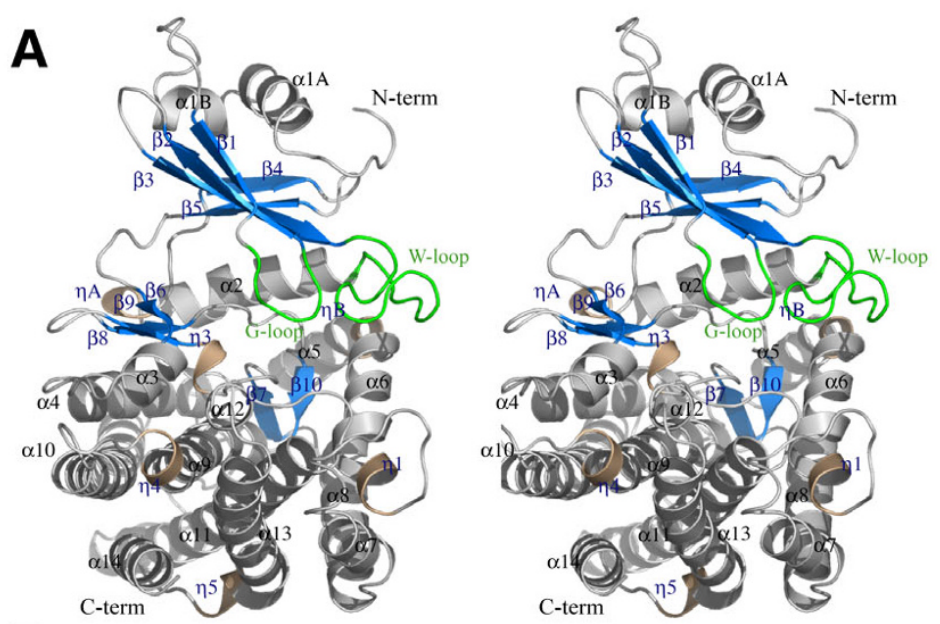

B
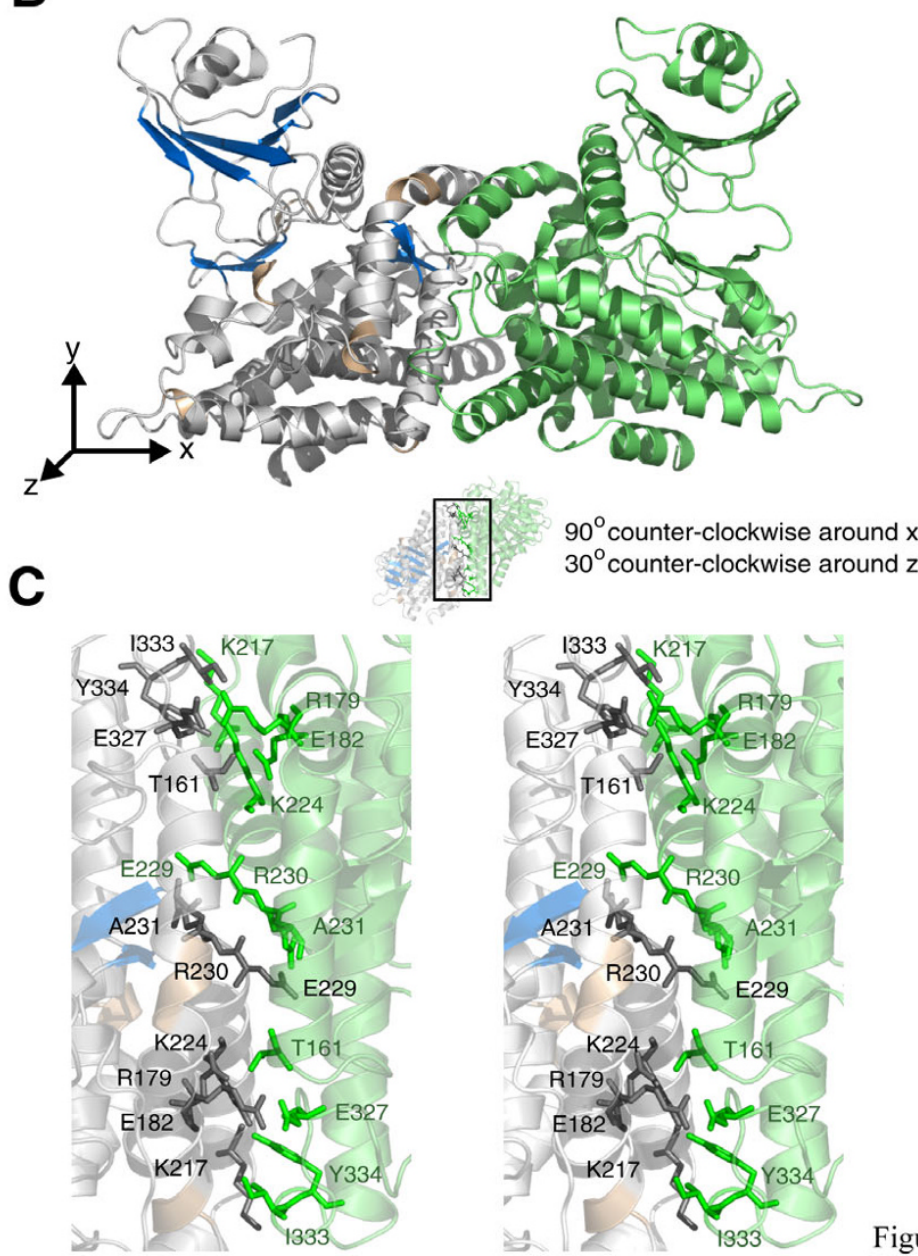

Figure 2

Figure 2

Structure of $A$. thaliana MTR kinase. (a) Stereo representation of the plant MTR kinase monomer. Monomer A of the MTRKADP-MTR complex is shown, with the nucleotide and substrate omitted. $\alpha$ helices are represented as grey coils with the $3_{10}$ helices as wheat coils. $\beta$ strands are represented as blue arrows and loops as grey tubes. The G-loop and W-loop are coloured in green. (b) Dimeric structure of the complex with monomer A coloured as in (a) and monomer B in green. (c) Stereo representation of detailed interactions between the two monomers. Figure 2 was prepared using PyMOL [5I]. 
A
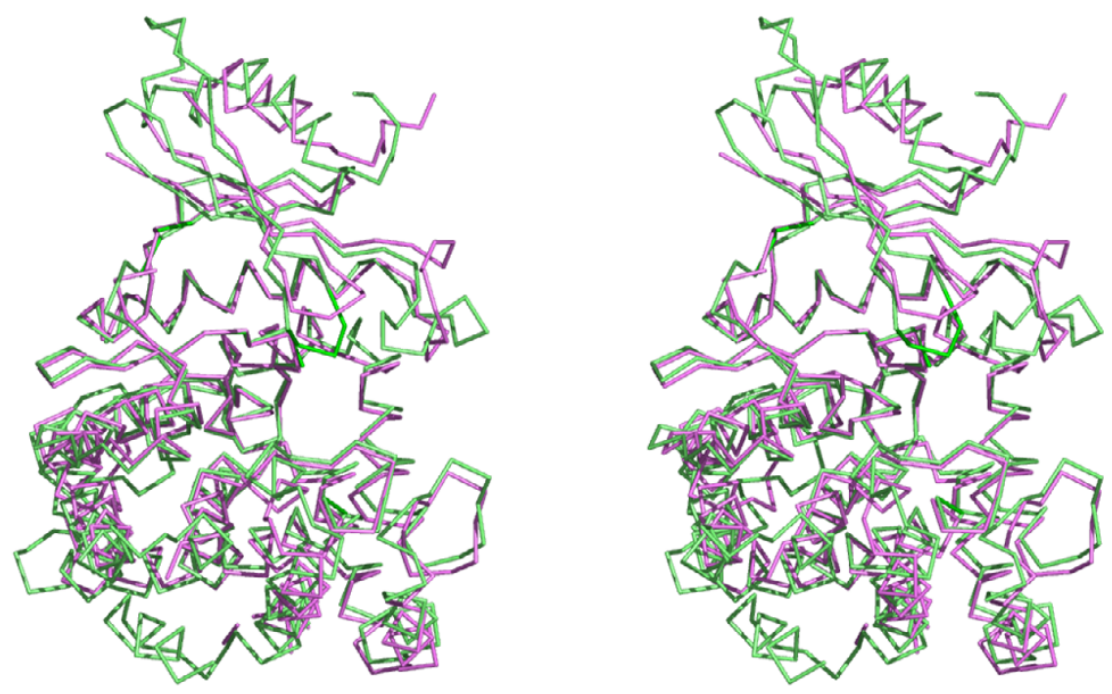

B

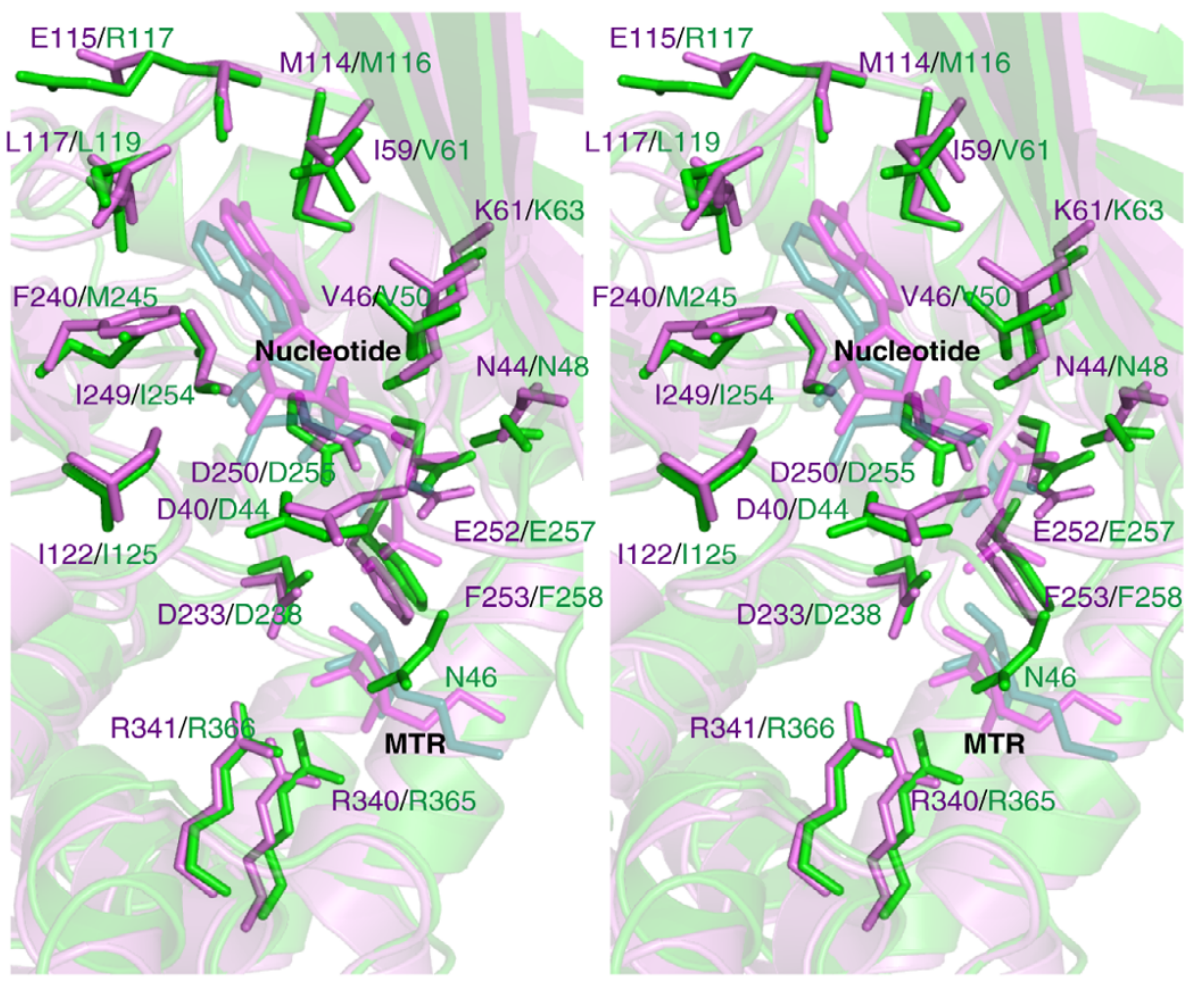

Figure 3

\section{Figure 3}

(a) Stereo representation of $C^{\alpha}$ superimposition of $B$. subtilis MTR kinase AMPPCP-MTR complex (PDB: 2 PUN) in violet and A. thaliana MTR kinase ADP-MTR complex (PDB: $\underline{2 P Y W}$ ) in green. (b) Stereo stick presentation of the active sites of the $B$. subtilis MTR kinase in violet and $A$. thaliana MTR kinase in green. The two structures have been superimposed as in (a) and are shown in transparent cartoon. Monomer A is shown. The nucleotides and substrate MTR are shown in transparent stick representation (and labelled) in order to show the residues behind. This figure was prepared using PyMOL [5I]. 
K224, E229, R230 and A231). Additional inter-subunit interactions are found at the N-terminus of helix $\alpha 5$ (T161), the C-terminus of helix $\alpha 6$ (R179 and E182), as well as the loop between helices $\alpha 10$ and $\alpha 11$ (E327, I333 and Y334) (Fig. 1). The interactions between these residues are depicted in Fig. 2c. Interestingly, when comparing the dimeric interfaces of the B. subtilis and A. thaliana enzymes, the identities of the residues involved in dimerization are not conserved (Fig. 1). The interactions are nevertheless concentrated in approximately the same region of the protein, especially the C-terminal end of helix $\alpha 8$, and result in exactly the same dimeric structure.

Although the tertiary structures of MTR kinase and other protein kinase-like phosphotransferases are highly similar, their quaternary structures are distinct. While MTR kinase dimerizes mainly via interactions between helices $\alpha 8$, choline kinase dimerizes via interactions between helices that are structurally equivalent to helices $\alpha 2$ of MTR kinase. Although crystal structures of APH show the protein as dimers $[28,29]$, they are not physiologically relevant as the enzyme is observed as a monomer in solution [30]. Rio2 protein kinase is also observed as a monomer [23].

\section{Nucleotide binding site}

The nucleotide binds in the cleft region between the two lobes of the protein. The adenine ring of the nucleotide binds near the linker region in a hydrophobic pocket. The N6 and N1 nitrogens of the adenine ring are hydrogenbonded to the backbone carbonyl oxygen of R117 and the amide nitrogen of L119, respectively (Fig. 4). The strictly conserved K63 also interacts with the $\alpha$-phosphoryl group of the nucleotide via an ionic interaction. A structurally conserved water molecule (W11 in monomer A; W12 in monomer B) donates two hydrogen bonds to the nucleotide: one to the $\mathrm{N} 7$ nitrogen of the adenine ring and the other to the $\mathrm{O} 1$ oxygen of the nucleotide's $\alpha$-phosphoryl group (Fig. 4). This water molecule is also observed in all of the bacterial MTR kinase nucleotide complex structures determined to date [26].

\section{Functionally important loops}

There are four conserved loop regions in MTR kinases: the G-loop between strands $\beta 1$ and $\beta 2$, the HGD catalytic loop between strand $\beta 7$ and the $3_{10}$ helix $\eta 3$, the $\mathrm{Mg}(\mathrm{II})$ binding DXE-motif between strands $\beta 9$ and $\beta 10$, and the semi-conserved W-loop between strand $\beta 3$ and helix $\alpha 2$ (Fig. 1). The G-loop of MTR kinases has a highly conserved GXGNXN motif (residues 43-48) and is structurally analogous the "nucleotide positioning loop" in APH(3')-IIIa [31] and the "Gly triad" GXGXXG motif found in many eukaryotic protein kinases.

\section{Gly-rich loop (G-loop) and solvent accessibility}

In A. thaliana MTR kinase, the G-loop plays a similar role in anchoring and coordinating the bound nucleotide. Residue D44 of the G-loop, although not absolutely conserved, makes a 2.6 Å hydrogen bond to the nucleotide's O3' ribosyl oxygen, while the backbone and terminal amide nitrogen's of $\mathrm{N} 48$ interact with the $\beta$-phosphoryl oxygens. The G-loop of A. thaliana MTR kinase has an extended conformation, with interactions formed between N46 and the O4 oxygen of MTR (Fig. 4b and 5a). This extended G-loop conformation is different from that observed in the bacterial MTR kinase, as the equivalent residue in the $B$. subtilis enzyme, N42, is over $10 \AA$ away from the $\mathrm{O} 4$ oxygen of MTR (Fig. 5d). In most of the bacterial structures determined to date [26], the G-loop is disordered, and in no case does it interact with MTR. A consequence of this extended G-loop in the Arabidopsis enzyme is that it shields the active site from solvent (Fig. 5a), a feature that more closely resembles a eukaryotic protein kinase active site than that of the B. subtilis MTR kinase (Fig. 5c). Since the G-loop appears to deny the entry and/or exit of nucleotides, conformational changes - loop movement at a minimum - will need to occur to allow nucleotide entry to the apo-form the enzyme.

\section{DXE-motif and $\mathbf{M g}$ binding}

In the A. thaliana MTR kinase structure, two $\mathrm{Mg}$ (II) ions assist in the binding of the ADP. Mg(II) ions are important for MTR kinase activity. The kinase activity is optimal in the presence of $\mathrm{Mg}$ (II) ions, attenuated to $20 \%$ in the presence of $\mathrm{Mn}$ (II) ions, and abolished in the presence of $\mathrm{Ca}(\mathrm{II})$ ions [32]. Residues D255 and E257 are part of the strictly conserved DXE-motif on the loop between strands $\beta 9$ and $\beta 10$ and are essential for $M g(I I)$ binding. The first magnesium ion, $\mathrm{Mg}(1)$, is chelated by the carboxyl $\mathrm{O}^{\delta}$ oxygens of D255, the carboxyl $\mathrm{O}^{\varepsilon}$ oxygen of E257, the $\mathrm{O} 2$ oxygen of the $\beta$-phosphoryl group of ADP, and two water molecules. These water molecules in turn interact with the O1 oxygen of MTR and the catalytic residue D238 (see below) (Fig. 4b). The second magnesium ion, $\operatorname{Mg}(2)$, is chelated by the $\alpha$ - and $\beta$-phosphoryl oxygens of ADP, the $\mathrm{O}^{\delta}$ oxygen of D255 and three water molecules. One of the water molecules that interacts with $\mathrm{Mg}(2)$ (W3 in monomer A and W6 in monomer B) also interacts with $\mathrm{O} 1$ oxygen of MTR (Fig. 4b). Unlike the bacterial MTR kinase where the water molecules surrounding the $\mathrm{Mg}$ (II) ions have poor electron density, both $\mathrm{Mg}$ (II) ions in the A. thaliana ADP-enzyme complex are coordinated in the most energetically favorable octahedral geometry.

Examination of amino acid sequences reveals that for all MTR kinases, except those from Nocardioides, Streptomyces and Clostridium species, the DXE-motif can be extended to a longer IDXEF motif (Fig. 1). The DXE-motif is always followed by a phenylalanine, F258 in A. thaliana MTR 


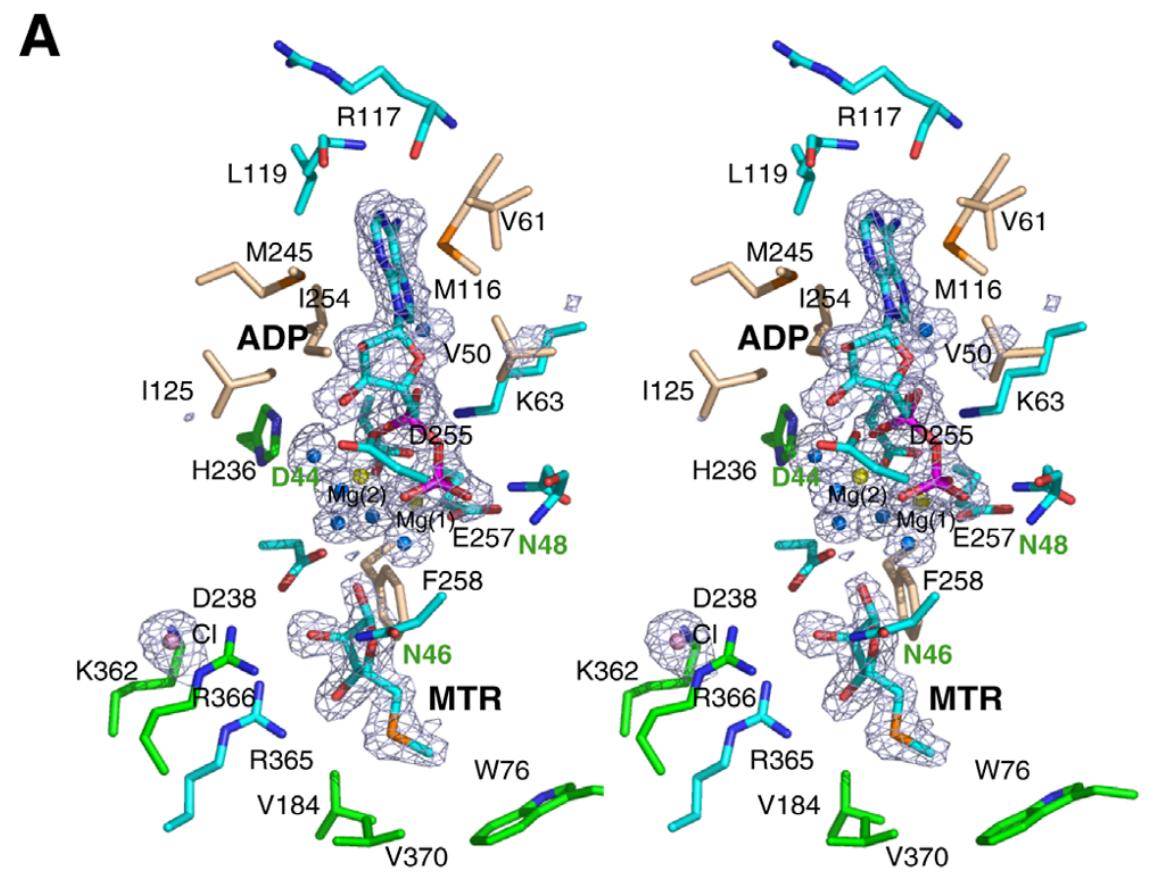

B

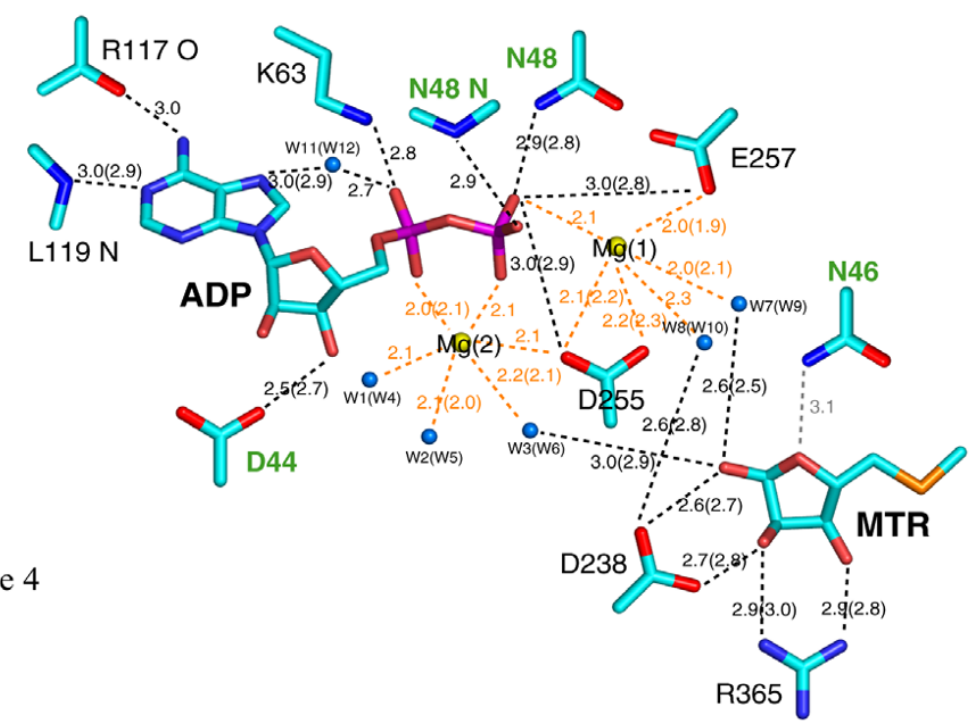

\section{Figure 4}

The active site of $A$. thaliana MTR kinase. (a) Stereo representation of the active site. The product ADP and the substrate MTR and nearby interacting residues are drawn in stick representation with $\mathrm{C}, \mathrm{N}, \mathrm{O}, \mathrm{P}$ and $\mathrm{S}$ atoms coloured in cyan, blue, red, magenta, and orange, respectively. Hydrophobic residues are shown with their $C$ atoms coloured in wheat. Residues that do not directly interact with ADP or MTR but are discussed in the text are shown with their $C$ atoms coloured in green. The magnesium ions, chloride ion and water molecules are shown as yellow, violet and blue spheres, respectively. The $\sigma_{\mathrm{A}}$-weighted

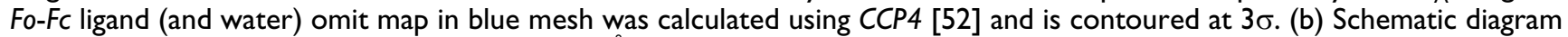
showing the hydrophilic interactions less than $3 \AA$ between the ligands and the protein. The colour schemes of the atoms are as described in Panel (a). The distances in $\AA$ between the ligands and the protein are for monomer $A$ while those for monomer $B$ are in parentheses. If the two distances are the same, only one value is shown. The distances around the two octahedrally coordinated $\mathrm{Mg}(\mathrm{II})$ ions are labelled in orange. Residues from the $\mathrm{G}$-loop are labelled in green. The distance between the $\mathrm{N} \delta$ amide nitrogen of N46 in the G-loop and the O4 of MTR is greater than $3 \AA$ and hence is shown in grey instead of black. Both panels were prepared using PyMOL [5I]. 

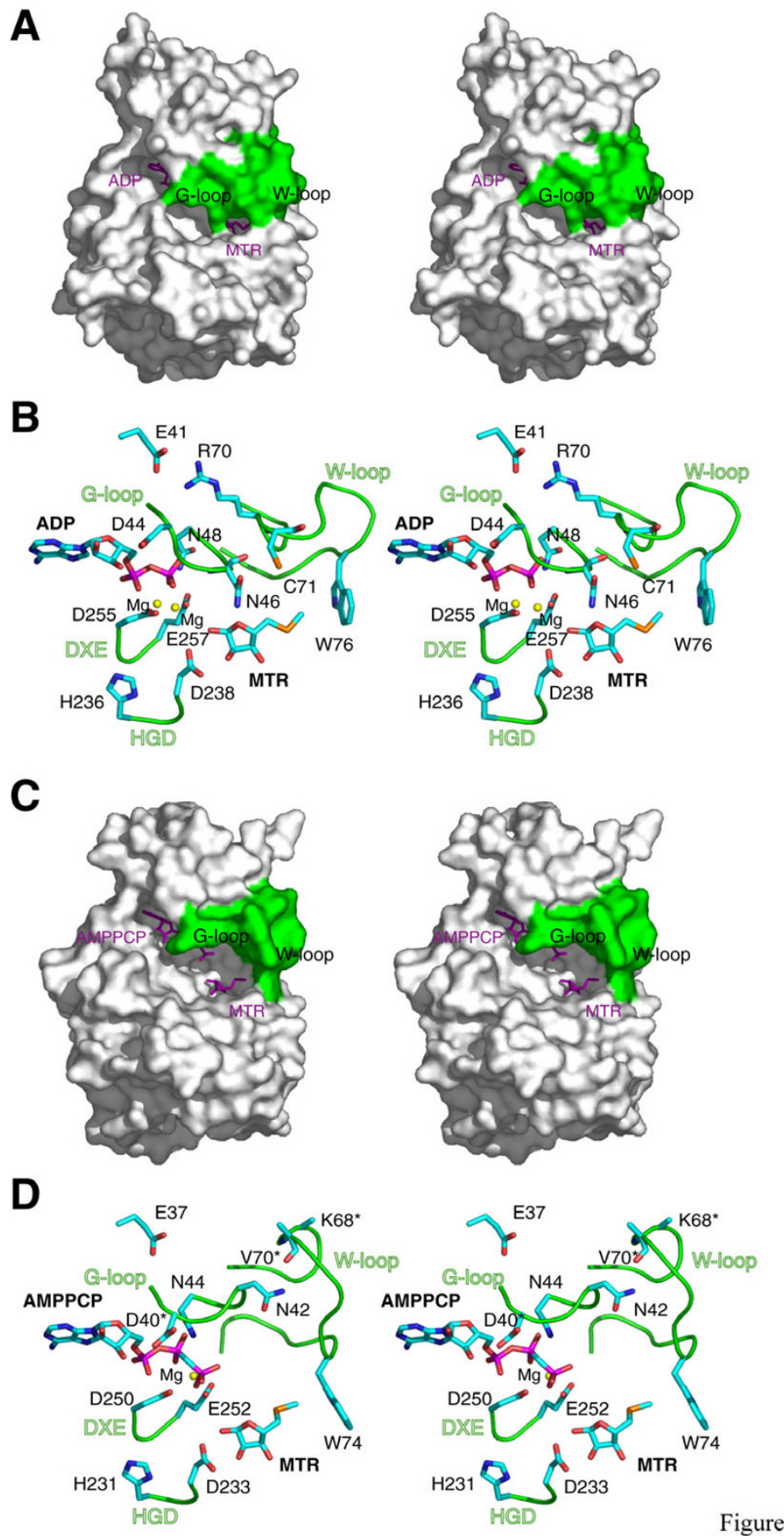

\section{Figure 5}

Comparison of the nucleotide binding pockets and loop conformations. Stereo surface representation of the A. thaliana MTR kinase ADP-MTR complex and B. subtilis MTR kinase AMPPCP-MTR complex are shown in panel (a) and (c), respectively with the G- and W-loop coloured in green and the ligands shown as purple sticks. Stereo cartoon representations of the four functionally important loops, the G-loop, the W-loop, the Mg-binding DXE-motif, and the HGD catalytic loop, found in the A. thaliana and B. subtilis enzymes are shown in (b) and (d), respectively. Substrates and important residues discussed in the text are shown in stick presentation with the same colour scheme as in Figure 4b. Residues labelled with an asterisk indicate that disordered side chains are observed in at least one subunit of all known structures of the $B$. subtilis enzyme. This figure was prepared using PyMOL [5I]. 
kinase, which is involved in MTR binding (see below). The significance of the DXE-motif is that this consensus sequence is analogous to the well defined DFG-motif that marks the start of the "activation loop" in protein kinases [33]. Unlike other phosphotransferases such as APH(3')IIIa or CAMP-dependent protein kinase (PKA), which use the aspartate in the DFG-motif (e.g. D208 in APH(3')-IIIa and D184 in PKA) and a second asparagine (e.g. N195 in APH(3')-IIIa and N171 in PKA) from the C-lobe to chelate the divalent metal ions, MTR kinase uses both acidic residues in the DXE-motif to bind $\mathrm{Mg}(\mathrm{II})$.

\section{RR-motif in the MTR binding site}

The substrate MTR is located beneath the nucleotide in the active site. In A. thaliana MTR kinase, MTR is bound in the higher energy $\alpha$-ribofuranose anomer with the substrate's $\mathrm{O} 1$ hydroxyl cis to the $\mathrm{O} 2$ and $\mathrm{O} 3$ hydroxyl groups (Fig. 4a). MTR is also observed in the same $\alpha$-anomer in the bacterial enzyme-substrate complex [26] (Fig. 3b). In the plant enzyme, the $\alpha$-conformation is stabilized by interactions with D238 and R365 as well as F258, which interacts with the substrates hydrophobic face (Fig. 4b). The O1 and $\mathrm{O} 2$ oxygens of MTR form hydrogen bonds ( 2.7 $\mathrm{A}$ ) with the carboxyl oxygens of D238 of the strictly conserved HGD-motif; while the $\mathrm{O} 2$ and $\mathrm{O} 3$ oxygens of MTR forms heteronuclear hydrogen bonds $(\sim 2.9 \AA)$ with the guanidium nitrogens of R365 of the strictly conserved twin arginine RR-motif (Fig. 1). The second arginine R366 does not interact directly with MTR but may facilitate the release of the anionic product, MTR 1-P. In the A. thaliana enzyme, a lysine (K362) is located near the RR-motif (Fig. $4 \mathrm{a})$, and to mitigate the electrostatic repulsion between these three positively charged residues, a chloride ion is found interacting with K362.

Interestingly, examination of the multiple sequence alignment (Fig. 1) shows that the residue equivalent to K362 in the $A$. thaliana enzyme, three residues upstream of the RRmotif, is always either a lysine or a glutamate residue in all known sequences of MTR kinases. Most bacterial MTR kinases including the well-studied Klebsiella pneumoniae and all of the Bacillus enzymes, have a glutamate at this position. This glutamate will help neutralize the positive charge imposed by the RR-motif in the MTR binding site. A lysine residue near the RR-motif, as found in the plant A. thaliana, Oryza sativa (rice), Vitis vinifera (grape), and nitrogen-fixing bacteria Bradyrhizobium japonicum and Wolinella succinogenes, would perturb MTR binding by charge repulsion when the $\mathrm{pH}$ falls below the pKa of lysine. Thus, the K362 in A. thaliana MTR kinase could potentially explain why this enzyme's in vitro activity is optimal at $\mathrm{pH} 9$, near the $\mathrm{pK}_{\mathrm{a}}$ of lysine, while the optimal activity for K. pneumoniae MTR kinase occurs at pH 7 (Reference [34] and see Additional file 1).

\section{W-loop and MTR binding specificity}

The W-loop between strand $\beta 3$ and helix $\alpha 2$, named after the semi-conserved tryptophan (W) at the tip of this loop, appears to modulate the size of chemical group at the 5position that can fit into the MTR binding site. In A. thaliana MTR kinase, the 5-methylthio moiety of the MTR appears to be capped by $\mathrm{W} 76$ of the $\mathrm{W}$-loop and residues V184 and V370 from the C-lobe (Fig. 4a). These three residues would appear to restrict the length of the alkylthio moiety at the 5-position. Although these three residues are not strictly conserved across MTR kinase sequences, they are structurally and functionally equivalent to residues W74 of the W-loop, and L180 and L345 in B. subtilis MTR kinase. However, many MTR analogs with bulky extensions at the 5-position have been shown to be substrates and/or inhibitors of MTR kinase [11,35], although the bulkiness of the substitution does not correlate with their inhibitory concentrations [36]. In fact, these inhibitors have comparable $\mathrm{K}_{\mathrm{m}}$ values (in the $\mu \mathrm{M}$ range) to the native substrate in many bacterial and plant MTR kinases $[15,32,33]$. Moreover, comparison between A. thaliana and $B$. subtilis MTR kinase shows that both the 5-methylthio moiety of MTR and the W-loop adopt different conformations in the otherwise comparable overall active sites (Fig. 3b and 5). These results suggest that the W-loop needs to be flexible to allow the entry of different sized MTR analogs to the active site. This hypothesis is consistent with the observation in B. subtilis MTR kinase structures that the density of the W-loop is often disordered [26], implying its flexibility.

\section{Conformational variations}

In the structural alignment of bacterial and plant MTR kinase, the bound ADP in A. thaliana is shifted toward the C-lobe when compared to the nucleotide in the B. subtilis enzyme (Fig. 3b). This observation raises the question whether the plant MTR kinase has a more closed conformation between the two-lobes than its bacterial homolog. Although the overall structural alignment of the two enzymes shows no major conformation changes, a minor rigid body shift of 1-1.5 $\AA$ in the N-lobe becomes apparent when only the C-lobes of the two structures are aligned. However, this displacement does not definitively demonstrate a potential inter-lobal movement in MTR kinase on substrate binding, because the intrinsic variation resulting from the sequence variation between the two enzymes - even neglecting the intrinsic structure coordinate errors of both structures - is more than $1.5 \AA$. In other words, a $1.5 \AA$ displacement observed between the N-lobes is within the expected structural variation of two non-identical protein sequences [37].

Despite the low overall $\mathrm{C}^{\alpha}$ RMSD between the structures of A. thaliana and B. subtilis MTR kinase, the conformations of the G- and W-loop in the two enzymes differ by 
3.8 $\AA$ and $5.3 \AA$ in their $\mathrm{C}^{\alpha}$ RMSDs, respectively (Fig. 5b and $5 \mathrm{~d}$ ). This observation is striking as the sequence identity of the 6-residue G-loop between the two enzymes is $100 \%$, and that of the 16-residue W loop is $50 \%$ (Fig. 1 ). These RMSD values are close to the expected values if two random loops of the same length are taken from the Protein Data Bank and structurally aligned [38]. The conformational variations between the two enzymes are the result of different interactions outside the loop regions. In the A. thaliana enzyme, the highly conserved E41, preceding the G-loop, interacts with R70 of the W-loop via an ionic interaction (Fig. 5b). The corresponding E37 and K68 in the B. subtilis enzyme do not interact (Fig. 5d). Moreover, in the plant enzyme, the main chain carbonyl oxygen of N46 (G-loop) forms a hydrogen bond to the main chain nitrogen of $\mathrm{C} 71$ (W-loop) (Fig. 5b), but in the bacterial enzyme, the corresponding N42 (G-loop) interacts with main chain carbonyl oxygen of V70 (W-loop) via the side chain $\mathrm{N}^{\delta}$ nitrogen instead (Fig. $5 \mathrm{~d}$ ). The difference in loop conformations could also be due to the differences in how they interact with the substrate in the homologous active sites (Fig. 3b). Until an apo-form of $A$. thaliana MTR kinase becomes available, it would be difficult to determine whether the variations in the loop conformations are substrate induced.

\section{ATPase activity in MTR kinase}

The A. thaliana MTR kinase complex was prepared by cocrystallization with excess but equal amount of ATP $\gamma$ S and MTR, but surprisingly, ADP and MTR were found in the active site. Thus, consistent with the soaking and co-crystallization experiments of bacterial MTRK in the presence of ATP [26], the plant enzyme appears to be able to hydrolyze the weakly hydrolysable ATP $\gamma$ S to ADP. Without extensive kinetic characterization it is hard to know whether this ATP hydrolysis is coupled to phosphorylation of MTR, because while only MTR was found in the active site, it might bind preferentially to MTR 1-P. The observation that $A$. thaliana MTR kinase, like its bacterial homologue, may have considerable ATPase activity in the absence of the substrate MTR will have an impact on the strategies used to design herbicides that target the enzyme.

\section{Conclusion}

The structure of a eukaryotic MTR kinase has been determined for the first time. Although the enzyme has been refolded into its active state, it has comparable tertiary and quaternary structures to its bacterial homolog. The active site is comparable between the two enzymes with the DXE-motif coordinating the nucleotide- $\mathrm{Mg}$, the D238 of the HGD catalytic loop polarizing the MTR O1 oxygen, and the RR-motif interacting with MTR. The G-loop and W-loop of the A. thaliana MTR kinase adopt different conformations, a consequence potentially of differences in their microenvironments. Unlike its bacterial homolog, the G-loop of A. thaliana MTR kinase has an extended conformation, which shields most of the active site from solvent, a feature that resembles a eukaryotic protein kinase more closely than the bacterial enzyme. The A. thaliana MTR kinase has optimal activity at higher $\mathrm{pH}$ than its bacterial homolog and this can be explained by the presence a lysine, rather than a glutamate, near the RR-motif in MTR binding site. The structure of A. thaliana MTR kinase also provides a template for structure-based design of agrochemicals - particularly herbicides whose effectiveness could be regulated by nutrient levels. Generic protein kinase inhibitors likely inhibit MTR kinase as they inhibit the remotely homologous APH enzymes [39], but these kinase inhibitors are often associated with complex steps of synthesis and isolation and high cost. The strictly conserved RR-motif in MTR binding site along with the nearby catalytic aspartate offers an opportunity for a simpler organic salt of an MTR analog to specifically inhibit MTR kinase at lower cost.

\section{Methods}

A. thaliana MTR kinase was cloned, and expressed in E. coli, and purified from inclusion bodies as a linear peptide, refolded into its active form and co-crystallized with substrates as described previously [25]. The effect of $\mathrm{pH}$ on MTR kinase activity was examined using a modification of the standard enzyme assay [34] in which the imidazole buffer (pH 6.5) was replaced by $100 \mathrm{mM}$ sodium phosphate ( $\mathrm{pH} 4-9.5)$. Assays used $300 \mathrm{ng}$ of recombinant enzyme and were performed in triplicate. Although prior to co-crystallization the enzyme was incubated with ATP $\gamma$ S and MTR, only ADP and MTR were found in the resulting structure. The crystals belong to space group $C 2(a=162.5 \AA, b=83.2 \AA, c=91.1 \AA, \beta=$ $117.8^{\circ}$ ) with a dimer per asymmetric unit. Prior to data collection the crystal was soaked in a solution containing $25 \%$ (v/v) ethylene glycol, 7.5\% (w/v) PEG8000, $75 \mathrm{mM}$ sodium cacodylate $\mathrm{pH}$ 6.5, and $0.15 \mathrm{M}$ magnesium acetate for 5 seconds and flash frozen in a nitrogen cryostream. Data were collected to $1.9 \AA$ A resolution at Station X8C, National Synchrotron Light Source, Brookhaven National Laboratory, and processed using $d^{*}$ TREK [40] and the DREAR program package [41].

The structure of $A$. thaliana MTR kinase was determined by molecular replacement using the program Phaser [42-44] and the B. subtilis enzyme as the search model [45]. Cycles of structure refinement were then alternated between Refmac [46] and model building using Coot [47]. The final structural model of $A$. thaliana MTR kinase has $\mathrm{R} / \mathrm{R}_{\text {free }}$ factors of $16.3 \% / 19.7 \%$. Both monomers in the asymmetric unit have bound ADP and MTR molecules as well as two magnesium and one chloride ions. An additional magnesium ion was found to participate in crystal packing, and a total of five ethylene glycol molecules and 778 water 
molecules have been modeled in the asymmetric unit. A. thaliana MTR kinase has 420 residues. Residues 1-2 and 420 of monomer A, and 1, 30-36 and 420 of monomer B could not be modeled due to poor or missing electron density. The side chains of residues 32, 288-289, and 380 of monomer A, and residues 2-5, 56, 418 of monomer B were also truncated to alanine due to poor electron density. The data, refinement and structure validation statistics are summarized in Table 1.

\section{List of abbreviations}

ADP: adenosine 5'-diphosphate; MTR: 5-methylthioribose; PDB: Protein Data Bank; SAM: S-adenosyl-Lmethionine; ATP: adenosine 5'-triphosphate; SAH: S-adenosylhomocysteine; MTA: 5'-methylthioadenosine; MTR 1-P: 5-methylthioribose 1-phosphate; APH(3')-IIIa: 3',5"aminogylcoside phosphotransferase type IIIa; cAMP: cyclic adenosine monophosphate; PKA: protein kinase A; RMSD: root mean square deviation; ATP $\gamma$ S: adenosine 5'( $\beta, \gamma$-thio)triphosphate; FAD: flavin adenine dinucleotide; $\mathrm{NAD}(\mathrm{P}) \mathrm{H}$ : nicotinamide adenine dinucleotide (phosphate); AMPPCP: $\beta, \gamma$-methyleneadenosine 5'-triphosphosphate.

\section{Coordinate}

The coordinates of the A. thaliana MTR kinase in complex with ADP and MTR have been deposited in the Protein Data Bank, accession number 2PYW.

\section{Authors' contributions}

S-YK - sub-cloning, expression, purification, refolding, crystallization, data collection, structure determination and analysis, and writing the manuscript. KAC - cloning, expression, kinetic analysis, and synthesis of MTR. PLH concept, analysis, writing and finalizing the manuscript. All authors have read and approved the final manuscript.

\section{Additional material}

\section{Additional file 1}

Maximum activity versus $p H$. Percent maximum activity versus $p H$ for $\mathrm{A}$. thaliana (red) and K. pneumoniae (blue) MTR kinase. The A. thaliana MTR kinase activity was measured as decribed in the Material and Methods, the results for $\mathrm{K}$. pneumoniae are from reference [34].

Click here for file

[http://www.biomedcentral.com/content/supplementary/14726807-7-70-S1.pdf]

Table I: Diffraction Data and Crystallographic Refinement Statistics

\begin{tabular}{|c|c|}
\hline Space Group & $C 2$ \\
\hline Cell dimensions $\left(\AA,^{\circ}\right)$ & $a=162.5, b=82.2, c=91.1 \beta=117.8$ \\
\hline Wavelength $(\AA)$ & 1.0 \\
\hline Resolution $(\AA)$ & $80.6-1.9$ \\
\hline Average redundancy ${ }^{a}$ & $3.4(2.9)$ \\
\hline$R_{\text {merge }}$ b $(\%)^{a}$ & $4.5(17.5)$ \\
\hline Completeness (\%) ${ }^{\mathrm{a}}$ & $98.7(90.8)$ \\
\hline$<\left.|>| \sigma\right|^{a}$ & $16.9(4.8)$ \\
\hline Number of reflections (working/test) & $78352 / 4105$ \\
\hline Number of protein atoms/heteroatoms ${ }^{c}$ & $6648 / 103$ \\
\hline Number of water molecules & 778 \\
\hline$R_{\text {cryst }} / R_{\text {free }}(\%)^{d}$ & $16.3 / 19.7$ \\
\hline Bond length $(\AA)$ & 0.014 \\
\hline Bond angle $\left({ }^{\circ}\right)$ & 1.4 \\
\hline \multicolumn{2}{|l|}{ Average $B$ factor $\left(\AA^{2}\right)$} \\
\hline Proteins & 16.4 \\
\hline Ligands (ADP and MTR) & 16.4 \\
\hline Magnesium ions & 18.0 \\
\hline Chloride ions & 20.7 \\
\hline Water molecules & 25.3 \\
\hline \multicolumn{2}{|l|}{ Ramachandran Plote } \\
\hline Total Favoured (\%) & 97.8 \\
\hline Total Allowed (\%) & 100 \\
\hline DPIf coordinate error based on $R_{\text {free }}(\AA)$ & 0.13 \\
\hline
\end{tabular}

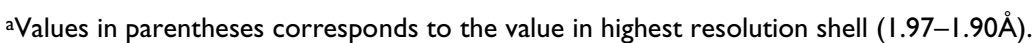

${ }^{b} R_{\text {merge }}=\sum \sum|l(k)-<|>\mid / \sum I(k)$ where I (k) and $<\mid>$ represent the diffraction intensity values of the individual measurements and the corresponding mean values. The summation is over all unique measurements.

cHeteroatoms include ADP, MTR, ethylene glycol, magnesium and chloride ions.

${ }^{d R_{\text {cryst }}}=\sum\left|F_{\text {obs }}-F_{\text {calc }}\right| / \sum|F o b s| ; R_{\text {free }}$ is $R_{\text {cryst }}$ for the $5 \%$ cross validated test data.

eAs defined by the Ramachandran plot in MolProbity [53].

fCruickshank's diffraction component precision index (DPI) [54] as an estimate of coordinate error. 


\section{Acknowledgements}

This work is supported by research grants from the Canada Institutes of Health Research (\#43998), the United States Department of Agriculture (\#02-0047), Idaho INBRE program (NIH NCRR P20RR0I6464), W.M. Keck Foundation and the Lucille P. Markey Charitable Trust. P.L.H. is the recipient of a Canada Research Chair. S.-Y.K. has been funded in part by postgraduate scholarships from the Natural Sciences and Engineering Research Council of Canada, the Ontario Student Opportunities Trust Fund and The Hospital for Sick Children Foundation Student Scholarship Program. Station X8-C is supported by the United States Department of Energy, and Multi-User Resource grants from the Canadian Institutes of Health Research and the Natural Sciences and Engineering Research Council of Canada.

\section{References}

I. Roje S: S-Adenosyl-I-methionine: Beyond the universal methyl group donor. Phytochemistry 2006.

2. Clarke S, Banfield K: S-adenosylmethionine-dependent methyltransferase. In Homocysteine in Health and Disease Edited by: Carmel $\mathrm{R}$, Jacobsen DW. New York , Cambridge University Press; 2001:63-78.

3. Pegg AE: Polyamine metabolism and its importance in neoplastic growth and a target for chemotherapy. Cancer Res 1988, 48(4):759-774.

4. Sauter M, Lorbiecke R, Ouyang B, Pochapsky TC, Rzewuski G: The immediate-early ethylene response gene OsARDI encodes an acireductone dioxygenase involved in recycling of the ethylene precursor S-adenosylmethionine. Plant J 2005, 44(5):718-729.

5. Bleecker $A B$, Kende $H$ : Ethylene: a gaseous signal molecule in plants. Annu Rev Cell Dev Biol 2000, I6: I-I8.

6. Imai A, Matsuyama T, Hanzawa Y, Akiyama T, Tamaoki M, Saji H, Shirano Y, Kato T, Hayashi H, Shibata D, Tabata S, Komeda Y, Takahashi T: Spermidine synthase genes are essential for survival of Arabidopsis. Plant Physiol 2004, I 35(3): I 565-I 573.

7. Kasukabe Y, He L, Nada K, Misawa S, Ihara I, Tachibana S: Overexpression of spermidine synthase enhances tolerance to multiple environmental stresses and up-regulates the expression of various stress-regulated genes in transgenic Arabidopsis thaliana. Plant Cell Physiol 2004, 45(6):71 2-722.

8. Miyazaki JH, Yang SF: Metabolism of 5-Methylthioribose to Methionine. Plant Physiol 1987, 84(2):277-28I.

9. Sekowska A, Denervaud V, Ashida H, Michoud K, Haas D, Yokota A, Danchin $A$ : Bacterial variations on the methionine salvage pathway. BMC Microbiol 2004, 4(I):9.

10. Pegg AE, Williams-Ashman HG: Phosphate-stimulated breakdown of 5'-methylthioadenosine by rat ventral prostate. Biochem J 1969, I I 5(2):24I-247.

II. Riscoe MK, Ferro AJ, Fitchen JH: Analogs of 5-methylthioribose, a novel class of antiprotozoal agents. Antimicrob Agents Chemother 1988, 32(12): 1904-1906.

12. Gianotti AJ, Tower PA, Sheley JH, Conte PA, Spiro C, Ferro AJ, Fitchen JH, Riscoe MK: Selective killing of Klebsiella pneumoniae by 5-trifluoromethylthioribose. Chemotherapeutic exploitation of the enzyme 5-methylthioribose kinase. J Biol Chem 1990, 265(2):831-837.

13. Singh V, Evans GB, Lenz DH, Mason JM, Clinch K, Mee S, Painter GF, Tyler PC, Furneaux RH, Lee JE, Howell PL, Schramm VL: Femtomolar transition state analogue inhibitors of 5'-methylthioadenosine/S-adenosylhomocysteine nucleosidase from Escherichia coli. J Biol Chem 2005, 280( I 8): I8265-I8273.

14. Lee JE, Singh V, Evans GB, Tyler PC, Furneaux RH, Cornell KA, Riscoe MK, Schramm VL, Howell PL: Structural rationale for the affinity of pico- and femtomolar transition state analogues of Escherichia coli 5'-methylthioadenosine/S-adenosylhomocysteine nucleosidase. J Biol Chem 2005, 280( I 8): |8274-|8282.

15. Sauter M, Cornell KA, Beszteri S, Rzewuski G: Functional analysis of methylthioribose kinase genes in plants. Plant Physiol 2004, I 36(4):406 I-407I.

16. Sekowska A, Mulard L, Krogh S, Tse JK, Danchin A: MtnK, methylthioribose kinase, is a starvation-induced protein in Bacillus subtilis. BMC Microbiol 200 I, I(I): I5.
17. Tower PA, Johnson LL, Ferro AJ, Fitchen JH, Riscoe MK: Synergistic activity of 5-trifluoromethylthioribose and inhibitors of methionine synthesis against Klebsiella pneumoniae. Antimicrob Agents Chemother 199I, 35(8): I557-I56I.

18. Tower PA, Alexander DB, Johnson LL, Riscoe MK: Regulation of methylthioribose kinase by methionine in Klebsiella pneumoniae. J Gen Microbiol 1993, I39(5): I027-I03I.

19. Walter MW: Structure-based design of agrochemicals. Nat Prod Rep 2002, I9(3):278-29I.

20. Holm L, Sander C: Protein structure comparison by alignment of distance matrices. J Mol Biol 1993, 233(I): I23-138.

21. Peisach D, Gee $P$, Kent $C, X u Z$ : The crystal structure of choline kinase reveals a eukaryotic protein kinase fold. Structure 2003, I I (6):703-7|3.

22. Burk DL, Hon WC, Leung AK, Berghuis AM: Structural analyses of nucleotide binding to an aminoglycoside phosphotransferase. Biochemistry 200I, 40(30):8756-8764.

23. LaRonde-LeBlanc N, Wlodawer A: Crystal structure of A. fulgidus Rio2 defines a new family of serine protein kinases. Structure 2004, I 2(9): I585- I594.

24. Fraternali F, Cavallo L: Parameter optimized surfaces (POPS): analysis of key interactions and conformational changes in the ribosome. Nucleic Acids Res 2002, 30( I 3):2950-2960.

25. Ku SY, Yip P, Cornell KA, Riscoe MK, Howell PL: Crystallization and preliminary $X$-ray analysis of 5 '-methylthioribose kinase from Bacillus subtilis and Arabidopsis thaliana. Acta Crystallogr D Biol Crystallogr 2004, 60(Pt I): I I6-I I9.

26. Ku SY, Yip P, Cornell KA, Riscoe MK, Behr JB, Guillerm G, Howell PL: Structures of 5-methylthioribose kinase reveal substrate specificity and unusual mode of nucleotide binding. J Biol Chem 2007, 282(30):22195-22206.

27. Guranowski A: Plant 5-methylthioribose kinase. Plant Physiol 1983, 71:932-935.

28. Hon WC, McKay GA, Thompson PR, Sweet RM, Yang DS, Wright GD, Berghuis AM: Structure of an enzyme required for aminoglycoside antibiotic resistance reveals homology to eukaryotic protein kinases. Cell I997, 89(6):887-895.

29. Nurizzo D, Shewry SC, Perlin MH, Brown SA, Dholakia JN, Fuchs RL, Deva T, Baker EN, Smith CA: The crystal structure of aminoglycoside-3'-phosphotransferase-Ila, an enzyme responsible for antibiotic resistance. J Mol Biol 2003, 327(2):49I-506.

30. Ganelin VL, Demina AS, Petiushenko RM, Sazykin lu O, Navashin SM: [Aminoglycoside-3'-phosphotransferase from Actinomyces fradiae. Its isolation, purification and properties]. Antibiotiki 1979, 24(6):424-430.

31. Thompson PR, Boehr DD, Berghuis AM, Wright GD: Mechanism of aminoglycoside antibiotic kinase APH(3')-IIla: role of the nucleotide positioning loop. Biochemistry 2002 , 4 I (22):700 I-7007.

32. Ferro AJ, Marchitto KS: 5-Methylthioribose kinase (Enterobacter aerogenes). Methods Enzymol 1983, 94:36I-364.

33. Kannan N, Neuwald AF: Did protein kinase regulatory mechanisms evolve through elaboration of a simple structural component? J Mol Biol 2005, 35 I(5):956-972.

34. Cornell KA, Winter RW, Tower PA, Riscoe MK: Affinity purification of 5-methylthioribose kinase and 5-methylthioadenosine/S-adenosylhomocysteine nucleosidase from Klebsiella pneumoniae [corrected]. Biochem J 1996, 3 I 7 ( Pt I):285-290.

35. Ferro AJ, Barrett A, Shapiro SK: 5-Methylthioribose kinase. A new enzyme involved in the formation of methionine from 5 methylthioribose. J Biol Chem 1978, 253(I 7):6021-6025.

36. Guillerm G: Personal Communication. 2005.

37. Chothia C, Lesk AM: The relation between the divergence of sequence and structure in proteins. Embo J 1986, 5(4):823-826.

38. Fernandez-Fuentes N, Fiser A: Saturating representation of loop conformational fragments in structure databanks. BMC Struct Biol 2006, 6:15.

39. Daigle DM, McKay GA, Wright GD: Inhibition of aminoglycoside antibiotic resistance enzymes by protein kinase inhibitors. $J$ Biol Chem 1997, 272(40):24755-24758.

40. Pflugrath JW: The finer things in X-ray diffraction data collection. Acta Crystallogr D Biol Crystallogr 1999, 55(Pt I0): |7| 8-1725.

41. Blessing RH: Dream - Data Reduction and Error Analysis Routines for Accurate Single-Crystal Diffraction Intensity Measurements. Journal of Applied Crystallography 1986, I 9:4 I 2-4 I 2. 
42. Read RJ: Pushing the boundaries of molecular replacement with maximum likelihood. Acta Crystallogr D Biol Crystallogr 200 I, 57(Pt I 0): | 373- | 382 .

43. Storoni LC, McCoy AJ, Read RJ: Likelihood-enhanced fast rotation functions. Acta Crystallogr D Biol Crystallogr 2004, 60(Pt 3):432-438.

44. McCoy AJ, Grosse-Kunstleve RW, Storoni LC, Read RJ: Likelihoodenhanced fast translation functions. Acta Crystallogr D Biol Crystallogr 2005, 6 I (Pt 4):458-464.

45. Ku SY, Smith GD, Howell PL: ADP-2Ho as a phasing tool for nucleotide-containing proteins. Acta Crystallogr D Biol Crystallogr 2007, 63:493-499.

46. Murshudov GN: Refinement of macromolecular structures by the maximum-likelihood method. Acta Crystallogr D Biol Crystallogr 1997, 53(Pt 3):240-255.

47. Emsley P, Cowtan K: Coot: model-building tools for molecular graphics. Acta Crystallogr D Biol Crystallogr 2004, 60(Pt I2 Pt I):2126-2I32.

48. Kabsch W, Sander C: Dictionary of protein secondary structure: pattern recognition of hydrogen-bonded and geometrical features. Biopolymers 1983, 22(12):2577-2637.

49. Notredame C, Higgins DG, Heringa J: T-Coffee: A novel method for fast and accurate multiple sequence alignment. J Mol Biol 2000, 302(I):205-217.

50. Gouet P, Courcelle E, Stuart DI, Metoz F: ESPript: analysis of multiple sequence alignments in PostScript. Bioinformatics 1999, I 5(4):305-308.

5I. Delano WL: The PyMOL molecular graphics system. Edited by: Scientific D. San Carlos, CA, USA, DeLano Scientific; 2002.

52. Collaborative Computational Project N: The CCP4 suite: programs for protein crystallography. Acta Crystallogr D Biol Crystallogr 1994, 50(Pt 5):760-763.

53. Lovell SC, Davis IW, Arendall WB 3rd, de Bakker PI, Word JM, Prisant MG, Richardson JS, Richardson DC: Structure validation by Calpha geometry: phi,psi and Cbeta deviation. Proteins 2003, 50(3):437-450.

54. Cruickshank DW: Remarks about protein structure precision. Acta Crystallogr D Biol Crystallogr 1999, 55 (Pt 3):583-60I. http.//www.biomedcentral.com/1472-6807/7/70 